Jpn. J. Phys. Fitness Sports Med. 2006, 55 Suppl. : S263 \$ 268

\title{
THE OPTIMUM INTENSITY AND MODE OF COOLING DOWN PROCEDURES AFTER POST REPETITIVE INTERMITTENT HIGH-INTENSITY EXERCISE
}

\author{
Kenichi Suijo ${ }^{1}$, Yoshiharu FujIEdA ${ }^{2)}$, RYOHEI ISAKU ${ }^{1)}$, \\ HisATAKA AmBe ${ }^{1)}$, KAZUKi Sumi ${ }^{2)}$, Shota YASUKAWA ${ }^{2)}$, \\ TAKUYA SEKIYA $^{1)}$ and MASASHI KAWAGUCHI ${ }^{1}$ )
}

\begin{abstract}
The purpose of this study was to compare the effects derived from different modes of active cooling down procedures post repetitive intermittent high-intensity exercise. Seven Japanese male swimmers underwent repetitive 3 bouts of Wingate anaerobic tests on the $1^{\text {st }}$ day and another Wingate on the following $2^{\text {nd }}$ day. In each 3 intervals and post all the Wingate testing on the $2^{\text {nd }}$ day, the participants performed a cooling down randomly chosen from 3 different intensities of 10-min bike-riding and a 10-min static stretching. As to muscle power outputs and emotional conditions, the optimum cooling down intensities were observed at $80 \%$ ventilatoly threshold (VT) after the $1^{\text {st }}$ set and $60 \%$ VT level after the $2^{\text {nd }}$ set. Effective intensities of blood lactate removal rate were observed at $60 \%$ and $80 \%$ VT level. Thus, the optimum cooling down post repetitive intermittent high-intensity exercise could be dynamic bike-riding at $60 \%$ or $80 \%$ VT level.

(Jpn. J. Phys. Fitness Sports Med. 2006, 55 Suppl : S263 \$268)

key word : Cooling down, Wingate anaerobic test, Muscle power output, Emotional condition, Lactate removal rate
\end{abstract}

\section{Introduction}

It has been suggested that when active recovery is applied between repeated bouts, it may facilitate performance more than when applying at passive rest ${ }^{1}$. When active recovery is applied during the rest interval between short-duration sprints in cycling, performance is better maintained compared to passive recovery ${ }^{2}$ ). This strong point of active recovery may be attributed to increased muscle blood flow, which in turn may facilitate oxygen supply to the cell for a faster phosphocreatine ( $\mathrm{PCr}$ ) resynthesis. During successive bouts of sprint exercise, a short-duration interval period leads to incomplete resynthesis of $\mathrm{PCr}^{3,4)}$. Anaerobic glycolysis results in high levels of muscle lactate associated with hydrogen. Lactate can no longer be considered the cause of fatigue ${ }^{5)}$. However, intramuscular metabolic byproducts, such as inorganic phosphate as well as hydrogen ions, may act to impair muscle function. Even though aerobic ATP contribution increases to com- pensate for the reduced rate of ATP provided by anaerobic sources ${ }^{3)}$, it does not prevent a significant reduction in power output and velocity which occurs during repeated short-duration cycling, swimming or running. In addition, exercise performance may often depend upon emotional conditions ${ }^{6)}$.

In this study, we investigated the physiological and emotional effects derived from different mode of active cooling down procedures, which in turn can be warming up for the next bout at the same time, post repetitive intermittent high-intensity exercise.

\section{Methods}

1. Subjects ; Seven Japanese male collegiate competitive swimmers (age ; $19.9 \pm 0.9$ years, height; $173.1 \pm 6.8 \mathrm{~cm}$, weight $; 63.6 \pm 8.3 \mathrm{~kg}$ ) agreed to participate in this study. All the subjects were fully informed in detail about the experimental procedures and any known risks, and then signed an informed consent statement. Participants had been trained in competitive swimming for at least 5 years.

\footnotetext{
${ }^{1)}$ Department of Health and Sport Sciences, Graduate School of Education, Tokyo Gakugei University
}

${ }^{2)}$ Department of Health and Sport Sciences, Tokyo Gakugei University 
2. Exercise procedures; Prior to the main trials, participants performed maximal cardio-pulmonary exercise testing on a cycle ergometer to evaluate the maximum oxygen uptake ( $\left.\dot{\mathrm{VO}}_{2} \max \right)$ and ventilatoly threshold (VT). Expired air was analyzed breathby-breath technique using breathing gas analyzer. VT point was determined by mean of $\dot{\mathrm{VE}} / \mathrm{VO}_{2}$ and $\dot{\mathrm{V} E} / \dot{\mathrm{VO}}_{2}$ trendgrams. The main trials consisted of repetitive 3 bouts of Wingate anaerobic tests on the $1^{\text {st }}$ day and another bout of Wingate on the following $2^{\text {nd }}$ day. Followed by each Wingate testing with 5-min passive recovery, every participant performed a 10-min cooling down exercise, which was randomly chosen from 3 different intensities of 10 -min bike-riding ( $60 \%, 80 \%$ and $100 \%$ of predetermined VT level) and a 10-min static stretching, and then rested in a sitting position for lasting 30-min. These four randomly assigned cooling down treat- ments were adopted on different days of at least 48-hour intervals. These protocols were shown in Figure 1.

Blood lactate concentrations $(\mathrm{mmol} / 1)$ were measured at 0,15, 25,45-min post Wingate test. Blood lactate removal rate was determined using the equation as follows;

(peak reading-reading at a point)/

$$
\text { ( peak reading-resting reading) } \times 100(\%)^{6} \text { ) }
$$

Profile of Mood States (POMS) was used to evaluate emotional conditions before and after 3 consecutive Wingate tests on the $1^{\text {st }}$ day and pre and post a single Wingate on the $2^{\text {nd }}$ day.

3. Statistical analysis; Results are expressed as means \pm standard deviations. Two-way analysis of variance (ANOVA) used to examine differences between means. The Tukey's post-hoc test was used to locate between different trials. The Wilcoxon's
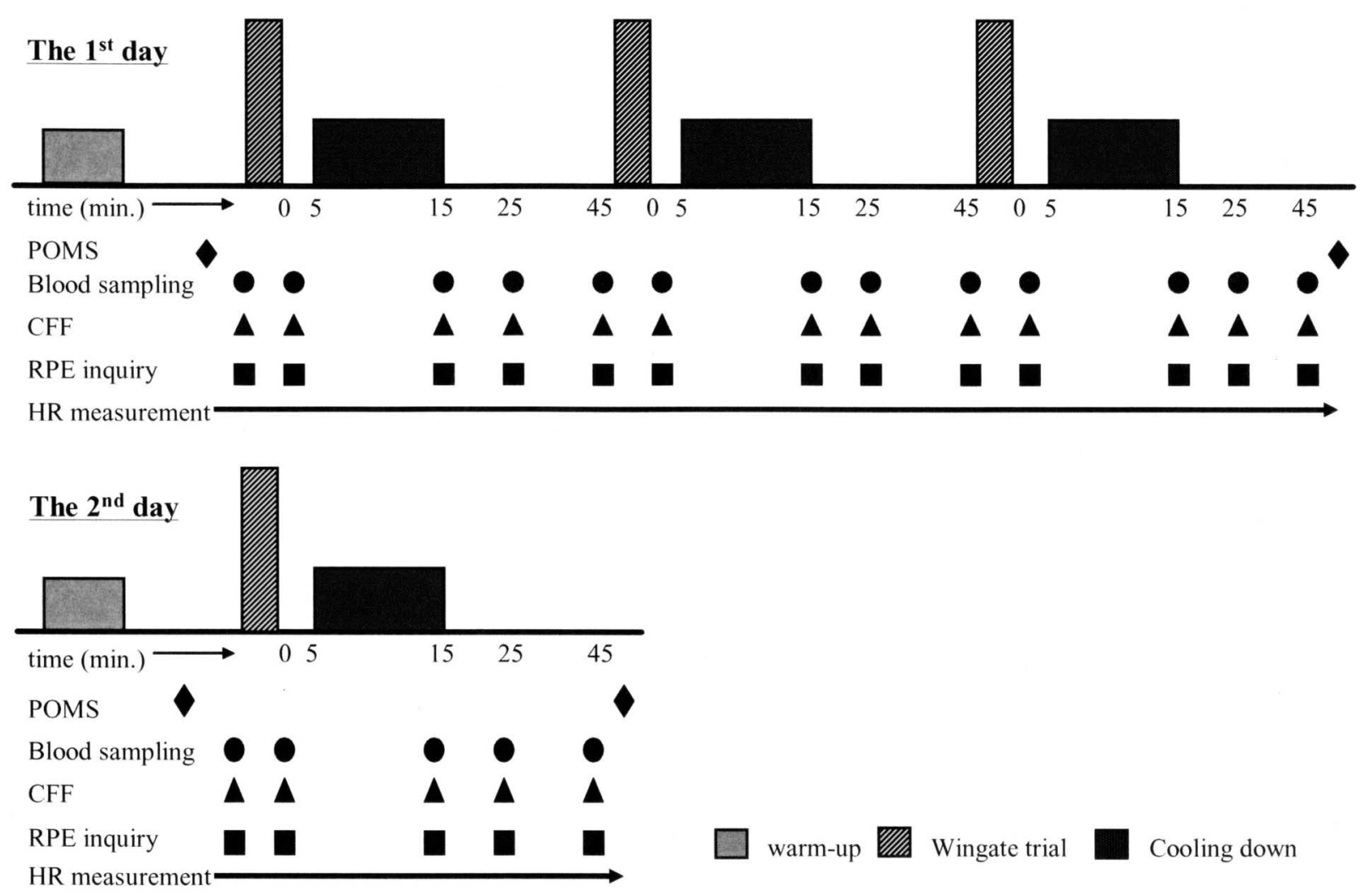

Fig. 1. Experiment protocol. POMS ; Profile of Mood States is often adopted to evaluate the acute changes in emotional aspects of Tension-Anxiety, Depression, Anger-Hostility, Fatigue, Vigor and Confusion under some kinds of interventions which includes exercise stresses. $\mathrm{CFF}$; Critical Flicker Fusion Frequency task assesses the integrative capacity of the central nervous system (CNS). CNS threshold is indicative of a reduction in the overall integrative activity of the CNS. 
signed rank test was adopted within the trial. Statistical significance was accepted $\mathrm{p}<0.05$ level.

\section{Results}

1. Power outputs in Wingate anaerobic test; Cooling down at the intensity of $80 \%$ VT level demonstrated significantly increased mean power output in the $2^{\text {nd }}$ trial $(566 \pm 83$ to $598 \pm 92$ watts; p $<0.05)$, while other 3 different ways did not. In the $3^{\text {rd }}$ trial of Wingate, only 60\% VT level cooling down maintained mean power output, while statistical decreases were found in $80 \%$ VT $(598 \pm 92$ to $584 \pm 92$ watts ; p $=0.09), 100 \% \operatorname{VT}(577 \pm 92$ to $564 \pm 102$

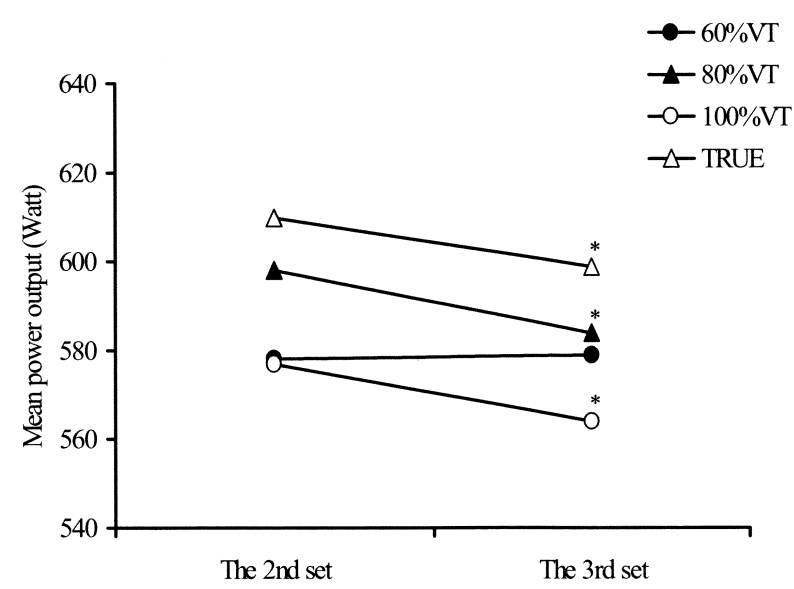

Fig. 2. Mean power output in the $2^{\text {nd }}$ and $3^{\text {rd }}$ set. TRUE ; TRUE STRETCH ${ }^{\mathrm{TM}}$ is an instrument for users to perform three-dimensionally static stretching. $*_{p}<0.05$ compared to the $2^{\text {nd }}$ set in cooling down at the intensity of $80 \%$ VT level, $100 \%$ VT level and TRUE.

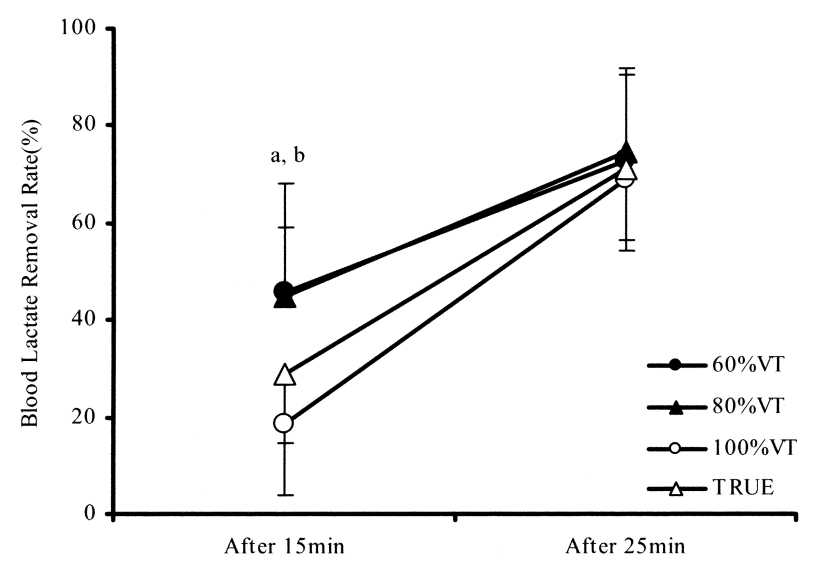

Fig. 3. Lactate removal rate in the $3^{\text {rd }}$ set. ${ }^{\mathrm{a}} \mathrm{p}<0.05$ between cooling down at the intensity of $60 \% \mathrm{VT}$ level vs. $100 \%$ VT. ${ }^{\mathrm{b}} \mathrm{p}<0.05$ between $80 \% \mathrm{VT}$ vs. $100 \%$ VT. watts $; \mathrm{p}<0.05)$ and static stretching procedures $(610 \pm 90$ to $599 \pm 86$ watts $; \mathrm{p}<0.05)$. These Wing ate outcomes in the $2^{\text {nd }}$ and the $3^{\text {rd }}$ trial were expressed in Figure 2.

2. Blood lactate removal rate; The blood lactate removal rate in $60 \% \mathrm{VT}$ intensity was higher than those in other cooling down procedures. Figure 3 illustrated that cooling down at the intensity of $100 \%$ VT demonstrated the most impaired lactate removal rate after the $3^{\text {rd }}$ Wingate performances, comparing with the rate at $60 \%$ VT $(100 \%$ VT vs. $60 \%$ $\mathrm{VT}=18.5 \pm 14.6 \%$ vs. $45.7 \pm 22.6 \% ; \mathrm{p}<0.05)$ and at $80 \% \mathrm{VT}$ levels $(100 \% \mathrm{VT}$ vs. $80 \% \mathrm{VT}=18.5 \pm$ $14.6 \%$ vs. $44.6 \pm 14.5 \% ; \mathrm{p}<0.05)$.

3. Profile of mood states (POMS); When the examiner informed the examinees that cooling down at $100 \%$ VT intensity would be applied, highly scored Tension-Anxiety was recognized prior to the initial Wingate trial on the $1^{\text {st }}$ day. Similarly the highest Depression score at $100 \%$ VT cooling down was found after the final $3^{\text {rd }}$ bout on the $1^{\text {st }}$ day $(100 \%$ $\mathrm{VT}$ vs. $60 \% \mathrm{VT}=53 \pm 6.8$ vs. $47 \pm 4.9 ; \mathrm{p}<0.05$ $100 \%$ VT vs. $80 \%$ VT $=53 \pm 6.8$ vs. $46 \pm 6.9 ; \mathrm{p}<$ $0.05,100 \%$ VT vs. static stretching $=53 \pm 6.8$ vs. 47 $\pm 4.0 ; \mathrm{p}<0.05)$. These emotional remarks were shown in Figure 4.

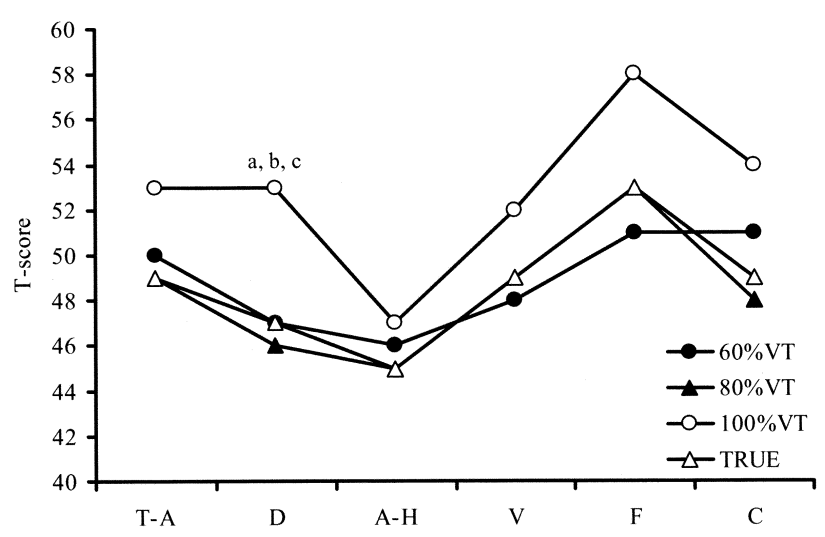

Fig. 4. POMS after the final $3^{\text {rd }}$ bout on the $1^{\text {st }}$ day. T-A ; Tension-Anxiety, D ; Depression, A-H ; Anger-Hostility, V ; Vigor, F ; Fatigue, C ; Confusion. ${ }^{\mathrm{a}} \mathrm{p}<0.05$ between cooling down at the intensity of $100 \%$ VT level vs. $60 \%$ VT. ${ }^{b}$ p $<0.05$ between $100 \%$ VT vs. $80 \%$ VT . ${ }^{c}$ p $<0.05$ between $100 \%$ VT vs. TRUE. 


\section{Discussion}

1. Power outputs in Wingate anaerobic test; The duration of the exercise as well as the rest interval separating successive sprints are crucial factors of performance maintenance ${ }^{7,8)}$. During a sprint lasting 10 to $30 \mathrm{~s}$, muscle lactate and hydrogen ion $\left(\mathrm{H}^{+}\right)$ concentrations reach high revels. Spriet et al. indicated that the increased concentration of $\mathrm{H}^{+}$may be responsible for the decreased performance during subsequent sprints, since it may impair the function of glycolytic enzymes ${ }^{9)}$. Westerblad et al. also described the increased accumulation of inorganic phosphate (Pi) may depress the muscular function by several mechanisms ${ }^{10)}$. These speculations could explain the decrement in mean power output observed in the $3^{\text {rd }}$ and $4^{\text {th }}$ Wingate trials especially during cooling down at the intensity of $100 \%$ VT level and static stretching procedures in this study.

2. Blood lactate removal rate; Iwahara et al. suggested that most effective intensity of blood lactate removal rates consisted in cooling down at the intensity of $80 \%$ to $100 \%$ lactate threshold level ${ }^{6)}$. In this study, however, the lactate removal rates impaired after cooling down exercise at 100\% VT level and in static stretching. More effective intensities of blood lactate removal rates, which can facilitate the following muscle performance, were observed at $60 \%$ VT and $80 \%$ VT level. The impaired rate at $100 \%$ VT level may be derived from blood lactate accumulation, since $100 \%$ VT level of cooling down after Wingate tests with only 5 -min resting itself was considered to be strenuous. With respect to static stretching procedure, insufficient milking actions by muscles, which does not wash-out accumulating lactate so much, could be responsible for lower lactate removal rate.

3. Profile of mood states (POMS); The muscle power output can be affected by emotional conditions. In other words, when a person has positive emotion toward a warm-up, it may enhance power output. To the contrary, it may be difficult to expect suitable effects when a person did not ${ }^{12)}$. Considered with such a psychological condition, the highest depression and higher Tension-Anxiety of negative mood states after being informed of a strenuous treatment of $100 \%$ VT intensity could be explained.

\section{Conclusion}

In this experimental model, the optimum cooling down post repetitive intermittent high-intensity exercise could be dynamic bike-riding at $60 \%$ or $80 \%$ VT level.

\section{References}

1) Maglischo E. Swimming fastest. Human kinetics. (2003), Champaign, III.

2) Bogdanis G. C., Nevill M. E., Lakomy H. K. A., Graham C. M. and Louis G. Effects of active recovery on power output during repeated maximal sprint cycling. Eur. J. Appl. Physiol.(1996), 74, 461-469.

3) Bogdanis G. C., Nevill M. E., A. Lakomy H. K. and Boobis L. H. Power output and muscle metabolism during and following recovery from 10 and $20 \mathrm{~s}$ of maximal sprint exercise in humans. Acta. Physiol. Scand.(1998), 163, 261-272.

4) Bogdanis G. C., Nevil M. E., Boobis L. H. and Lakomy H. K. A. Contribution of phosphocreatine and aerobic metbolism to energy supply during repeated sprint exercise. J. Appl. Physiol.(1996), 80(3), 876-884.

$5)$ Gladden L. B. Lactate metabolism: a new paradigm for the third millenium. J. Physiol.(2004), 558, 5-30.

6) Iwahara F., Ito M. and Asami T. Effect of cooling down on blood lactate removal and anaerobic workout in exhaustive cycle ergometer exercise. Jpn. J. Fitness Sports Med.(2003), 52, 499-512.

7) Balsom P., Seger J., Sjodin B. and Elkblom b. Physiological responses to maximal intensity intermittent exercise. Eur. J. Appl. Physiol.(1992), 65, 144-149.

8) Balsom P., Seger J., Sjodin B. and Elkblom b. Maximalintensity intemittent exercise: effect of recovery duration. Int. J. Sports Med.(1992), 13(7), 528-533.

9) Spriet L. L., Lindinger M. I., McKelvie R. S., Heigenhauser G. J. F. and Jones N. L. Mucsle glycogenolysis and $\mathrm{H}^{+}$concentration during maximal intermittent cycling. J. Appl. Physiol.(1989), 66, 8-13.

10) Westerblad A. and Allen D. Cellular mechanisms of skeltal muscle fatigue. Adv. Exp. Med. Biol.(2003), 538, 563-570.

11) Yamamoto M. and Yamamoto T. Effect of stretching, sport massage, light exercise and hot pack on recoveries of work capacity and blood lactate after strenuous exercise. Jpn. J. Fitness Sports Med.(1993), 42, 82-92.

12) Smith L. J. and Bozymowski M. F. Effect of attitude 
toward warm-ups on moter performance. Res. Qusrt. (1965), 36, 78-85. 